\title{
LOCATING $\mathfrak{A} x$, WHERE $\mathfrak{A}$ IS A SUBSPACE OF $\mathcal{B}(H)$
}

\author{
DOUGLAS S. BRIDGES
}

University of Canterbury, Christchurch, New Zealand

e-mail address: douglas.bridges@canterbury.ac.nz

\begin{abstract}
Given a linear space of operators on a Hilbert space, any vector in the latter determines a subspace of its images under all operators. We discuss, within a Bishop-style constructive framework, conditions under which the projection of the original Hilbert space onto the closure of the image space exists. We derive a general result that leads directly to both the open mapping theorem and our main theorem on the existence of the projection.
\end{abstract}

\section{INTRODUCTION}

Let $H$ be a real or complex Hilbert space, $\mathcal{B}(H)$ the space of bounded operators on $H$, and $\mathfrak{A}$ a linear subspace of $\mathcal{B}(H)$. For each $x \in H$ write

$$
\mathfrak{A} x \equiv\{A x: A \in \mathfrak{A}\},
$$

and, if it exists, denote the projection of $H$ onto the closure $\overline{\mathfrak{A} x}$ of $\mathfrak{A} x$ by [ $\mathfrak{A} x]$. Projections of this type play a very big part in the classical theory of operator algebras, in which context $\mathfrak{A}$ is normally a subalgebra of $\mathcal{B}(H)$; see, for example, [10, 11, 13, 15]. However, in the constructive 1$]$ setting - the one of this paper - we cannot even guarantee that $[\mathfrak{A} x]$ exists. Our aim is to give sufficient conditions on $\mathfrak{A}$ and $x$ under which $[\mathfrak{A} x]$ exists, or, equivalently, the set $\mathfrak{A} x$ is located, in the sense that

$$
\rho(v, \mathfrak{A} x) \equiv \inf \{\|v-A x\|: A \in \mathfrak{A}\}
$$

exists for each $v \in H$.

We require some background on operator topologies. Specifically, in addition to the standard uniform topology on $\mathcal{B}(H)$, we need

$\triangleright$ the strong operator topology: the weakest topology on $\mathcal{B}(H)$ with respect to which the mapping $T \rightsquigarrow T x$ is continuous for all $x \in H$;

$\triangleright$ the weak operator topology: the weakest topology on $\mathcal{B}(H)$ with respect to which the mapping $T \rightsquigarrow\langle T x, y\rangle$ is continuous for all $x, y \in H$.

2012 ACM CCS: [Theory of computation]: Constructive Mathematics.

2010 Mathematics Subject Classification: 03F60,46S30,47S30.

Key words and phrases: constructive, Hilbert space, space of operators, located.

${ }^{1}$ Our constructive setting is that of Bishop [2, 3, 6, in which the mathematics is developed with intuitionistic, not classical, logic, in a suitable set- or type-theoretic framework [1, 12] and with dependent choice permitted. 
These topologies are induced, respectively, by the seminorms of the form $T \rightsquigarrow\|T x\|$ with $x \in H$, and $T \rightsquigarrow|\langle T x, y\rangle|$ with $x, y \in H$. The unit ball 2

$$
\mathcal{B}_{1}(H) \equiv\{T \in \mathcal{B}(H):\|T\| \leqslant 1\}
$$

of $\mathcal{B}(H)$ is classically weak-operator compact, but constructively the most we can say is that it is weak-operator totally bounded (see [4]). The evidence so far suggests that in order to make progress when dealing constructively with a subspace or subalgebra $\mathfrak{A}$ of $\mathcal{B}(H)$, it makes sense to add the weak-operator total boundedness of

$$
\mathfrak{A}_{1} \equiv \mathfrak{A} \cap \mathcal{B}_{1}(H)
$$

to whatever other hypothesis we are making; in particular, it is known that $\mathfrak{A}_{1}$ is located in the strong operator topology — and hence $\mathfrak{A}_{1} x$ is located for each $x \in H$-if and only if it is weak-operator totally bounded [7, 14].

Recall that the metric complement of a subset $S$ of a metric space $X$ is the set $-S$ of those elements of $X$ that are bounded away from $X$. When $Y$ is a subspace of $X, y \in Y$, and $S \subset Y$, we define

$$
\rho_{Y}(y,-S) \equiv \inf \{\rho(y, z): z \in Y \cap-S\}
$$

if that infimum exists.

We now state our main result.

Theorem 1.1. Let $\mathfrak{A}$ be a uniformly closed subspace of $\mathcal{B}(H)$ such that $\mathfrak{A}_{1}$ is weak-operator totally bounded, and let $x$ be a point of $H$ such that $\mathfrak{A} x$ is closed and $\rho_{\mathfrak{A} x}\left(0,-\mathfrak{A}_{1} x\right)$ exists. Then the projection $[\mathfrak{A} x]$ exists.

Before proving this theorem, we discuss, in Section 2, some general results about the locatedness of sets like $\mathfrak{A} x$, and we derive, in Section 3, a generalisation of the open mapping theorem that leads to the proof of Theorem 1.1. Finally, we show, by means of a Brouwerian example, that the existence of $\rho_{\mathfrak{A} x}\left(0,-\mathfrak{A}_{1} x\right)$ cannot be dropped from the hypotheses of our main theorem.

\section{Some general LOCATEDness Results For $\mathfrak{A} x$}

We now prove an elementary, but helpful, result on locatedness in a Hilbert space.

Proposition 2.1. Let $\left(S_{n}\right)_{n \geqslant 1}$ be a sequence of located, convex subsets of a Hilbert space $H$ such that $S_{1} \subset S_{2} \subset \cdots$, let $S_{\infty}=\bigcup_{n \geqslant 1} S_{n}$, and let $x \in H$. For each $n$, let $x_{n} \in S_{n}$ satisfy $\left\|x-x_{n}\right\|<\rho\left(x, S_{n}\right)+2^{-n}$. Then

$$
\rho\left(x, S_{\infty}\right)=\inf _{n \geqslant 1} \rho\left(x, S_{n}\right)=\lim _{n \rightarrow \infty} \rho\left(x, S_{n}\right),
$$

in the sense that if any of these three numbers exists, then all three do and they are equal. Moreover, $\rho\left(x, S_{\infty}\right)$ exists if and only if $\left(x_{n}\right)_{n \geqslant 1}$ converges to a limit $x_{\infty} \in H$; in that case, $\rho\left(x, S_{\infty}\right)=\left\|x-x_{\infty}\right\|$, and $\|x-y\|>\left\|x-x_{\infty}\right\|$ for all $y \in S_{\infty}$ with $y \neq x_{\infty}$.

\footnotetext{
${ }^{2}$ Note that it is not constructively provable that every element $T$ of $\mathcal{B}(H)$ is normed, in the sense that the usual operator norm of $T$ exists. Nevertheless, when we write ' $\|T\| \leqslant 1$ ', we are using a shorthand for ' $\|T x\| \leqslant\|x\|$ for each $x \in H$ '. Likewise, ' $\|T\|<1$ ' means that there exists $c<1$ such that $\|T x\| \leqslant c\|x\|$ for each $x \in H$; and ' $\|T\|>1$ ' means that there exists $x \in H$ such that $\|T x\|>\|x\|$.
} 
Proof. Suppose that $\rho\left(x, S_{\infty}\right)$ exists. Then $\rho\left(x, S_{\infty}\right) \leqslant \rho\left(x, S_{n}\right)$ for each $n$. On the other hand, given $\varepsilon>0$ we can find $z \in S_{\infty}$ such that $\|x-z\|<\rho\left(x, S_{\infty}\right)+\varepsilon$. Pick $N$ such that $z \in S_{N}$. Then for all $n \geqslant N$,

$$
\rho\left(x, S_{\infty}\right) \leqslant \rho\left(x, S_{n}\right) \leqslant \rho\left(x, S_{N}\right) \leqslant\|x-z\|<\rho\left(x, S_{\infty}\right)+\varepsilon .
$$

The desired conclusion (2.1) now follows.

Next, observe that (by the parallelogram law in $H$ ) if $m \geqslant n$, then

$$
\begin{aligned}
\left\|x_{m}-x_{n}\right\|^{2} & \leqslant\left\|\left(x-x_{m}\right)-\left(x-x_{n}\right)\right\|^{2} \\
& =2\left\|x-x_{m}\right\|^{2}+2\left\|x-x_{n}\right\|^{2}-4\left\|x-\frac{1}{2}\left(x_{m}+x_{n}\right)\right\|^{2} \\
& \leqslant 2\left(\rho\left(x, S_{m}\right)+2^{-m}\right)^{2}+2\left(\rho\left(x, S_{n}\right)+2^{-n}\right)^{2}-4 \rho\left(x, S_{m}\right)^{2},
\end{aligned}
$$

since $\frac{1}{2}\left(x_{m}+x_{n}\right) \in S_{m}$. Thus

$$
\begin{aligned}
\left\|x_{m}-x_{n}\right\|^{2} \leqslant & 2\left(\left(\rho\left(x, S_{m}\right)+2^{-m}\right)^{2}-\rho\left(x, S_{m}\right)^{2}\right) \\
& +2\left(\left(\rho\left(x, S_{n}\right)+2^{-n}\right)^{2}-\rho\left(x, S_{m}\right)^{2}\right) .
\end{aligned}
$$

If $\rho\left(x, S_{\infty}\right)$ exists, then, by the first part of the proof, $\rho\left(x, S_{n}\right) \rightarrow \rho\left(x, S_{\infty}\right)$ as $n \rightarrow \infty$. It follows from this and (2.2) that $\left\|x_{m}-x_{n}\right\|^{2} \rightarrow 0$ as $m, n \rightarrow \infty$; whence $\left(x_{n}\right)_{n \geqslant 1}$ is a Cauchy sequence in $H$ and therefore converges to a limit $x_{\infty} \in \overline{S_{\infty}}$. Then

$$
\begin{aligned}
\rho\left(x, S_{\infty}\right) & =\rho\left(x, \overline{S_{\infty}}\right) \leqslant\left\|x-x_{\infty}\right\| \\
& =\lim _{n \rightarrow \infty}\left\|x-x_{n}\right\| \\
& \leqslant \lim _{n \rightarrow \infty}\left(\rho\left(x, S_{n}\right)+2^{-n}\right)=\rho\left(x, S_{\infty}\right) .
\end{aligned}
$$

Thus $\rho\left(x, S_{\infty}\right)=\left\|x-x_{\infty}\right\|$.

Conversely, suppose that $x_{\infty}=\lim _{n \rightarrow \infty} x_{n}$ exists. Let $0<\alpha<\beta$ and $\varepsilon=\frac{1}{3}(\beta-\alpha)$. Pick $N$ such that $2^{-N}<\varepsilon$ and $\left\|x_{\infty}-x_{n}\right\|<\varepsilon$ for all $n \geqslant N$. Either $\left\|x-x_{\infty}\right\|>\alpha+2 \varepsilon$ or $\left\|x-x_{\infty}\right\|<\beta$. In the first case, for all $n \geqslant N$,

$$
\begin{aligned}
\rho\left(x, S_{n}\right) & >\left\|x-x_{n}\right\|-2^{-n} \\
& \geqslant\left\|x-x_{\infty}\right\|-\left\|x_{\infty}-x_{n}\right\|-\varepsilon \\
& >(\alpha+2 \varepsilon)-\varepsilon-\varepsilon=\alpha .
\end{aligned}
$$

In the other case, there exists $\nu>N$ such that $\left\|x-x_{\nu}\right\|<\beta$; we then have

$$
\rho\left(x, S_{\nu}\right) \leqslant\left\|x-x_{\nu}\right\|<\beta .
$$

It follows from this and the constructive least-upper-bound principle ([6], Theorem 2.1.18) that

$$
\inf \left\{\rho\left(x, S_{n}\right): n \geqslant 1\right\}
$$

exists; whence, by (2.1),$d \equiv \rho\left(x, S_{\infty}\right)$ exists. 
Finally, suppose that $x_{\infty}$ exists, and consider any $y \in S_{\infty}$ with $y \neq x_{\infty}$. We have

$$
\begin{aligned}
0 & <\left\|y-x_{\infty}\right\|^{2}=\left\|y-x-\left(x_{\infty}-x\right)\right\|^{2} \\
& =2\|y-x\|^{2}+2\left\|x_{\infty}-x\right\|^{2}-4\left\|\frac{y+x_{\infty}}{2}-x\right\|^{2} \\
& =2\left(\|y-x\|^{2}-d^{2}\right)+2\left(\left\|x_{\infty}-x\right\|^{2}-d^{2}\right)=2\left(\|y-x\|^{2}-d^{2}\right),
\end{aligned}
$$

so $\|x-y\|>d$.

For each positive integer $n$ we write

$$
\mathfrak{A}_{n} \equiv n \mathfrak{A}_{1}=\left\{n A: A \in \mathfrak{A}_{1}\right\} .
$$

If $\mathfrak{A}_{1}$ is weak-operator totally bounded and hence strong-operator located, then $\mathfrak{A}_{n}$ has those two properties as well.

Our interest in Proposition 2.1 stems from this:

Corollary 2.2. Let $\mathfrak{A}$ be a linear subspace of $\mathcal{B}(H)$ with $\mathfrak{A}_{1}$ weak-operator totally bounded, and let $x, y \in H$. For each $n$, let $y_{n} \in \mathfrak{A}_{n}$ satisfy $\left\|y-y_{n}\right\|<\rho\left(x, \mathfrak{A}_{n} x\right)+2^{-n}$. Then

$$
\rho(y, \mathfrak{A} x)=\inf _{n \geqslant 1} \rho\left(y, \mathfrak{A}_{n} x\right)=\lim _{n \rightarrow \infty} \rho\left(y, \mathfrak{A}_{n} x\right) .
$$

Moreover, $\rho(y, \mathfrak{A} x)$ exists if and only if $\left(y_{n}\right)_{n \geqslant 1}$ converges to a limit $y_{\infty} \in H$; in which case, $\rho(y, \mathfrak{A} x)=\left\|y-y_{\infty}\right\|$, and $\|y-A x\|>\left\|y-y_{\infty}\right\|$ for each $A \in \mathfrak{A}$ such that $A x \neq y_{\infty}$.

One case of this corollary arises when the sequence $\left(\rho\left(y, \mathfrak{A}_{n} x\right)\right)_{n \geqslant 1}$ stabilises:

Proposition 2.3. Let $\mathfrak{A}$ be a linear subspace of $\mathcal{B}(H)$ such that $\mathfrak{A}_{1}$ is weak-operator totally bounded. Let $x, y \in H$, and suppose that for some positive integer $N, \rho\left(y, \mathfrak{A}_{N} x\right)=$ $\rho\left(y, \mathfrak{A}_{N+1} x\right)$. Then $\rho(y, \mathfrak{A} x)$ exists and equals $\rho\left(y, \mathfrak{A}_{N} x\right)$.

Proof. By Theorem 4.3.1 of [6], there exists a unique $z \in \overline{\mathfrak{A}_{N} x}$ such that $\rho\left(y, \mathfrak{A}_{N} x\right)=$ $\|y-z\|$. We prove that $y-z$ is orthogonal to $\mathfrak{A} x$. Let $A \in \mathfrak{A}$, and consider $\lambda \in \mathbf{C}$ so small that $\lambda A \in \mathfrak{A}_{1}$. Since,

we have

$$
z-\lambda A x \in \overline{\mathfrak{A}_{N+1} x}
$$

$$
\begin{aligned}
\langle y-z-\lambda A x, y-z-\lambda A x\rangle & \geqslant \rho\left(y, \mathfrak{A}_{N+1} x\right)^{2} \\
& =\rho\left(y, \mathfrak{A}_{N} x\right)^{2}=\langle y-z, y-z\rangle .
\end{aligned}
$$

This yields

$$
|\lambda|^{2}\|A x\|^{2}+2 \operatorname{Re}(\lambda\langle y-z, A x\rangle) \geqslant 0 .
$$

Suppose that $\operatorname{Re}\langle y-z, A x\rangle \neq 0$. Then by taking a sufficiently small real $\lambda$ with

$$
\lambda \operatorname{Re}\langle y-z, A x\rangle<0,
$$

we obtain a contradiction. Hence $\operatorname{Re}\langle y-z, A x\rangle=0$. Likewise, $\operatorname{Im}\langle y-z, A x\rangle=0$. Thus $\langle y-z, A x\rangle=0$. Since $A \in \mathfrak{A}$ is arbitrary, we conclude that $y-z$ is orthogonal to $\mathfrak{A} x$ and hence to $\overline{\mathfrak{A} x}$. It is well known that this implies that $z$ is the unique closest point to $y$ in the closed linear subspace $\overline{\mathfrak{A} x}$. Since $\mathfrak{A} x$ is dense in $\overline{\mathfrak{A} x}$, it readily follows that $\rho(y, \mathfrak{A} x)=\rho(y, \overline{\mathfrak{A} x})=\|y-z\|$. 
The final result in this section will be used in the proof of our main theorem.

Proposition 2.4. Let $\mathfrak{A}$ be a linear subspace of $\mathcal{B}(H)$ with weak-operator totally bounded unit ball, and let $x \in H$. Suppose that there exists $r>0$ such that

$$
\mathfrak{A}_{1} x \supset B_{\mathfrak{A} x}(0, r) \equiv \mathfrak{A} x \cap B(0, r) .
$$

Then $\mathfrak{A} x$ is located in $H$; in fact, for each $y \in H$, there exists a positive integer $N$ such that $\rho(y, \mathfrak{A} x)=\rho\left(y, \mathfrak{A}_{N} x\right)$.

Proof. Fixing $y \in H$, compute a positive integer $N>2\|y\| / r$. Let $A \in \mathfrak{A}$, and suppose that

$$
\|y-A x\|<\rho\left(y, \mathfrak{A}_{N} x\right) .
$$

We have either $\|A x\|<N r$ or $\|A x\|>2\|y\|$. In the first case, $N^{-1} A x \in B_{\mathfrak{A} x}(0, r)$, so there exists $B \in \mathfrak{A}_{1}$ with $N^{-1} A x=B x$ and therefore $A x=N B x$. But $N B \in \mathfrak{A}_{N}$, so

$$
\|y-A x\|=\|y-N B x\| \geqslant \rho\left(y, \mathfrak{A}_{N} x\right),
$$

a contradiction. In the case $\|A x\| \geqslant N r>2\|y\|$, we have

$$
\|y-A x\| \geqslant\|A x\|-\|y\|>\|y\| \geqslant \rho\left(y, \mathfrak{A}_{N} x\right),
$$

another contradiction. We conclude that $\|y-A x\| \geqslant \rho\left(y, \mathfrak{A}_{N} x\right)$ for each $A \in \mathfrak{A}$. On the other hand, given $\varepsilon>0$, we can find $A \in \mathfrak{A}_{N}$ such that $\|y-A x\|<\rho\left(y, \mathfrak{A}_{N} x\right)+\varepsilon$. It now follows that $\rho(y, \mathfrak{A} x)$ exists and equals $\rho\left(y, \mathfrak{A}_{N} x\right)$.

\section{Generalising the open mapping theorem}

The key to our main result on the existence of projections of the form $[\mathfrak{A} x]$ is a generalisation of the open mapping theorem from functional analysis ([6], Theorem 6.6.4). Before giving that generalisation, we note a proposition and a lemma.

Proposition 3.1. If $C$ is a balanced, convex subset of a normed space $X$, then $V \equiv \bigcup_{n \geqslant 1} n C$ is a linear subspace of $X$.

Proof. Let $x \in V$ and $\alpha \in \mathbf{C}$. Pick a positive integer $n$ and an element $c$ of $C$ such that $x=n c$. If $\alpha \neq 0$, then since $C$ is balanced, $|\alpha|^{-1} \alpha c \in C$, so

$$
\alpha x=\alpha n c=|\alpha| n|\alpha|^{-1} \alpha c \in|\alpha| n C \subset(1+|\alpha|) n C .
$$

In the general case, we can apply what we have just proved to show that

$$
(1+\alpha) x \in(1+|1+\alpha|) n C \subset(2+|\alpha|) n C .
$$

Now, since $C$ is balanced,

$$
-x=n(-c) \in n C \subset(2+|\alpha|) n C .
$$

Hence, by the convexity of $(2+|\alpha|) n C$,

$$
\alpha x=2 \frac{(1+\alpha) x-x}{2} \in 2(2+|\alpha|) n C .
$$

Taking $N$ as any integer $>2(2+|\alpha|) n$, we now see that $\alpha x \in N C \subset V$. In view of the foregoing and the fact that $(n C)_{n \geqslant 1}$ is an ascending sequence of sets, if $x^{\prime}$ also belongs to $V$ 
we can take $N$ large enough to ensure that $\alpha x$ and $x^{\prime}$ both belong to $N C$. Picking $c, c^{\prime} \in C$ such that $\alpha x=N c$ and $x^{\prime}=N c^{\prime}$, we obtain

$$
\alpha x+x^{\prime}=2 N\left(\frac{c+c^{\prime}}{2}\right) \in 2 N C,
$$

so $\alpha x+x^{\prime} \in V$.

We call a bounded subset $C$ of a Banach space $X$ superconvex if for each sequence $\left(x_{n}\right)_{n \geqslant 1}$ in $C$ and each sequence $\left(\lambda_{n}\right)_{n \geqslant 1}$ of nonnegative numbers such that $\sum_{n=1}^{\infty} \lambda_{n}$ converges to 1 and the series $\sum_{n=1}^{\infty} \lambda_{n} x_{n}$ converges, we have $\sum_{n=1}^{\infty} \lambda_{n} x_{n} \in C$. In that case, $C$ is clearly convex.

Lemma 3.2. Let $C$ be a located, bounded, balanced, and superconvex subset of a Banach space $X$, such that $X=\bigcup_{n \geqslant 1} n C$. Let $y \in X$ and $r>\|y\|$. Then there exists $\xi \in 2 C$ such that if $y \neq \xi$, then $\rho(z, C)>0$ for some $z$ with $\|z\|<r$.

Proof. Either $\rho(y, C)>0$ and we take $z=y$, or else, as we suppose, $\rho(y, C)<r / 2$. Choosing $x_{1} \in 2 C$ such that $\left\|y-\frac{1}{2} x_{1}\right\|<r / 2$ and therefore $\left\|2 y-x_{1}\right\|<r$, set $\lambda_{1}=0$. Then either $\rho\left(2 y-x_{1}, C\right)>0$ or $\rho\left(2 y-x_{1}, C\right)<r / 2$. In the first case, set $\lambda_{k}=1$ and $x_{k}=0$ for all $k \geqslant 2$. In the second case, pick $x_{2} \in 2 C$ such that $\left\|2 y-x_{1}-\frac{1}{2} x_{2}\right\|<r / 2$ and therefore $\left\|2^{2} y-2 x_{1}-x_{2}\right\|<r$, and set $\lambda_{2}=0$. Carrying on in this way, we construct a sequence $\left(x_{n}\right)_{n \geqslant 1}$ in $2 C$, and an increasing binary sequence $\left(\lambda_{n}\right)_{n \geqslant 1}$ with the following properties.

- If $\lambda_{n}=0$, then

$$
\rho\left(2^{n-1} y-\sum_{i=1}^{n} 2^{n-i-1} x_{i}, C\right)<\frac{r}{2}
$$

and

$$
\left\|2^{n} y-\sum_{i=1}^{n} 2^{n-i} x_{i}\right\|<r .
$$

- If $\lambda_{n}=1-\lambda_{n-1}$, then

$$
\rho\left(2^{n-1} y-\sum_{i=1}^{n} 2^{n-i-1} x_{i}, C\right)>0
$$

and $x_{k}=0$ for all $k \geqslant n$.

Compute $\alpha>0$ such that $\|x\|<\alpha$ for all $x \in 2 C$. Then the series $\sum_{i=1}^{\infty} 2^{-i} x_{i}$ converges, by comparison with $|\alpha| \sum_{i=1}^{\infty} 2^{-i}$, to a sum $\xi$ in the Banach space $X$. Since $\sum_{i=1}^{\infty} 2^{-i}=1$ and $C$ is superconvex, we see that

$$
\sum_{i=1}^{\infty} 2^{-i} x_{i}=2 \sum_{i=1}^{\infty} 2^{-i}\left(\frac{1}{2} x_{i}\right) \in 2 C .
$$

If $y \neq \xi$, then there exists $N$ such that

$$
\left\|y-\sum_{i=1}^{N} 2^{-i} x_{i}\right\|>2^{-N} r
$$


and therefore

$$
\left\|2^{N} y-\sum_{i=1}^{N} 2^{N-i} x_{i}\right\|>r .
$$

It follows that we cannot have $\lambda_{N}=0$, so $\lambda_{N}=1$ and therefore there exists $\nu \leqslant N$ such that $\lambda_{\nu}=1-\lambda_{\nu-1}$. Setting

$$
z \equiv 2^{\nu-1} y-\sum_{i=1}^{\nu-1} 2^{\nu-i-1} x_{i},
$$

we see that $\rho(z, C)>0$ and $\|z\|<r$, as required.

We now prove our generalisation of the open mapping theorem.

Theorem 3.3. Let $X$ be a Banach space, and $C$ a located, bounded, balanced, and superconvex subset of $X$ such that $\rho(0,-C)$ exists and $X=\bigcup_{n \geqslant 1} n C$. Then there exists $r>0$ such that $B(0, r) \subset C$.

Proof. Consider the identity

$$
X=\bigcup_{n \geqslant 1} \overline{n C} .
$$

By Theorem 6.6.1 of [6] (see also [8]), there exists $N$ such that the interior of $\overline{N C}$ is inhabited. Thus there exist $y_{0} \in N C$ and $R>0$ such that $B\left(y_{0}, R\right) \subset \overline{N C}$. Writing $y_{1}=N^{-1} y_{0}$ and $r=(2 N)^{-1} R$, we obtain $B\left(y_{1}, 2 r\right) \subset \bar{C}$.It follows from Lemma 6.6 .3 of [6] that $B(0,2 r) \subset \bar{C}$. Now consider any $y \in B(0,2 r)$. By Lemma 3.2, there exists $\xi \in 2 C$ such that if $y \neq \xi$, then there exists $z \in B(0,2 r)$ with $\rho(z, C)>0$. Since $B(0,2 r) \subset \bar{C}$, this is absurd. Hence $y=\xi \in 2 C$. It follows that $B(0,2 r) \subset 2 C$ and hence that $B(0, r) \subset C$.

Note that in Lemma 3.2 and Theorem 3.3 we can replace the superconvexity of $C$ by these two properties: $C$ is convex, and for each sequence $\left(x_{n}\right)_{n \geqslant 1}$ in $C$, if $\sum_{n=1}^{\infty} 2^{-n} x_{n}$ converges in $H$, then its sum belongs to $C$.

We now derive two corollaries of Theorem 3.3 .

Corollary 3.4 (The open mapping theorem ([6], Theorem 6.6.4) $\left.)^{3}\right)$. Let $X, Y$ be Banach spaces, and $T$ a sequentially continuous linear mapping of $X$ onto $Y$ such that $T(\overline{B(0,1)})$ is located and $\rho(0,-T(\overline{B(0,1)}))$ exists. Then there exists $r>0$ such that $B(0, r) \subset$ $T(\overline{B(0,1)})$.

Proof. In view of Theorem 3.3 , it will suffice to prove that $C \equiv T(\overline{B(0,1)})$ is superconvex. But if $\left(x_{n}\right)_{n \geqslant 1}$ is a sequence in $\overline{B(0,1)}$ and $\left(\lambda_{n}\right)_{n \geqslant 1}$ is a sequence of nonnegative numbers such that $\sum_{n=1}^{\infty} \lambda_{n}=1$, then $\left\|\lambda_{n} x_{n}\right\| \leqslant \lambda_{n}$ for each $n$, so $\sum_{n=1}^{\infty} \lambda_{n} x_{n}$ converges in $X$; moreover,

$$
\left\|\sum_{n=1}^{\infty} \lambda_{n} x_{n}\right\| \leqslant \sum_{n=1}^{\infty} \lambda_{n}=1,
$$

\footnotetext{
${ }^{3}$ This is but one version of the open mapping theorem; for another, see [5].
} 
so, by the sequential continuity of $T$,

$$
T\left(\sum_{n=1}^{\infty} \lambda_{n} x_{n}\right) \in C .
$$

Thus $C$ is superconvex.

Theorem 3.3 also leads to the proof of Theorem 1.1]

Proof. Taking $C \equiv \mathfrak{A}_{1} x$, we know that $C$ is located (since $\mathfrak{A}_{1}$ is weak-operator totally bounded and hence, by [7, 14, strong-operator located), as well as bounded and balanced. To prove that $C$ is superconvex, consider a sequence $\left(A_{n}\right)_{n \geqslant 1}$ in $\mathfrak{A}_{1}$, and a sequence $\left(\lambda_{n}\right)_{n \geqslant 1}$ of nonnegative numbers such that $\sum_{n=1}^{\infty} \lambda_{n}$ converges to 1 . For $k \geqslant j$ we have

$$
\left\|\sum_{n=j}^{k} \lambda_{n} A_{n}\right\| \leqslant \sum_{n=j}^{k} \lambda_{n},
$$

so $\sum_{n=1}^{\infty} \lambda_{n} A_{n}$ converges uniformly to an element $A$ of $\mathcal{B}_{1}(H)$. Since $\mathfrak{A}$ is uniformly closed, $A \in \mathfrak{A}_{1}$, so $\sum_{n=1}^{\infty} \lambda_{n} A_{n} x=A x \in \mathfrak{A}_{1} x$. Thus $C$ is superconvex. We can now apply Theorem 3.3. to produce $r>0$ such that $B_{\mathfrak{A} x}(0, r) \subset C$. The locatedness of $\mathfrak{A} x$, and the consequent existence of the projection $[\mathfrak{A} x]$, now follow from Proposition 2.4 .

We now discuss further the requirement, in Theorem 1.1, that $\rho_{\mathfrak{A} x}\left(0,-\mathfrak{A}_{1} x\right)$ exist, where $\mathfrak{A}_{1}$ is weak-operator totally bounded. We begin by giving conditions under which that requirement is satisfied.

If $\mathfrak{A} x$ has positive, finite dimension - in which case it is both closed and located in $H$-then $\mathfrak{A} x-\mathfrak{A}_{1} x$ is inhabited, so Proposition (1.5) of [9] can be applied to show that $\mathfrak{A} x-\mathfrak{A}_{1} x$ is located in $\mathfrak{A} x$. In particular, $\rho_{\mathfrak{A} x}\left(0,-\mathfrak{A}_{1} x\right)$ exists. On the other hand, if $P$ is a projection in $\mathcal{B}(H)$ and

$$
\mathfrak{A} \equiv\{P T P: T \in \mathcal{B}(H)\},
$$

then $\mathfrak{A}$ can be identified with $\mathcal{B}(P(H))$, so $\mathfrak{A}_{1}$ is weak-operator totally bounded. Moreover, if $x \neq 0$, then $\mathfrak{A} x=P(H)$ and so is both closed and located, $\mathfrak{A}_{1} x=\bar{B}(0,\|P x\|) \cap P(H)$, and $\rho_{\mathfrak{A} x}\left(0,-\mathfrak{A}_{1} x\right)=\|P x\|$.

We end with a Brouwerian example showing that we cannot drop the existence of $\rho_{\mathfrak{A} x}\left(0,-\mathfrak{A}_{1} x\right)$ from the hypotheses of Theorem 1.1. Consider the case where $H=\mathbf{R} \times \mathbf{R}$, and let $\mathfrak{A}$ be the linear subspace (actually an algebra) of $\mathcal{B}(H)$ comprising all matrices of the form

$$
T_{a, b} \equiv\left(\begin{array}{cc}
a & 0 \\
0 & b
\end{array}\right)
$$

with $a, b \in \mathbf{R}$. It is easy to show that $\mathfrak{A}$ is uniformly closed: if $\left(a_{n}\right),\left(b_{n}\right)$ are sequences in $\mathbf{R}$ such that $\left(T_{a_{n}, b_{n}}\right)_{n \geqslant 1}$ converges uniformly to an element $T \equiv\left(\begin{array}{cc}a_{\infty} & p \\ q & b_{\infty}\end{array}\right)$, then

$$
a_{n}=T_{a_{n}, b_{n}}\left(\begin{array}{l}
1 \\
0
\end{array}\right) \rightarrow T\left(\begin{array}{l}
1 \\
0
\end{array}\right)=a_{\infty},
$$

Likewise, $b_{n} \rightarrow b_{\infty}, p=0$, and $q=0$. Hence $T=T_{a_{\infty}, b_{\infty}} \in \mathfrak{A}$. 
Now, if $(x, y)$ is in the unit ball of $H$, then

$$
\begin{aligned}
\left\|T_{a, b}\left(\begin{array}{c}
x \\
y
\end{array}\right)\right\|^{2} & =\left\|\left(\begin{array}{c}
a x \\
b y
\end{array}\right)\right\|^{2}=a^{2} x^{2}+b^{2} y^{2} \\
& =a^{2}\left(x^{2}+y^{2}\right)+\left(b^{2}-a^{2}\right) y^{2} \\
& =a^{2}+\left(b^{2}-a^{2}\right) y^{2} .
\end{aligned}
$$

We see from this that if $a^{2} \geqslant b^{2}$, then $\left\|T_{a, b}\right\|^{2} \leqslant a^{2}$; moreover, $T_{a, b}(1,0)=a$, so $\left\|T_{a, b}\right\|^{2}=a^{2}$. If $a^{2}<b^{2}$, then a similar argument shows that $\left\|T_{a, b}\right\|^{2}=b^{2}$. It now follows that $\left\|T_{a, b}\right\|$ exists and equals $\max \{|a|,|b|\}$. Also, since, relative to the uniform topology on $\mathcal{B}(H), \mathfrak{A}_{1}$ is homeomorphic to the totally bounded subset

$$
\{(a, b): \max \{|a|,|b|\} \leqslant 1\}
$$

of $\mathbf{R}^{2}$, it is uniformly, and hence weak-operator, totally bounded.

Consider the vector $\xi \equiv(1, c)$, where $c \in \mathbf{R}$. If $c=0$, then $\mathfrak{A} \xi=\mathbf{R} \times\{0\}$, the projection of $H$ on $\mathfrak{A} \xi$ is just the projection on the $x$-axis, and $\rho((0,1), \mathfrak{A} \xi)=1$. If $c \neq 0$, then

$$
\mathfrak{A} \xi=\{(a, c b): a, b \in \mathbf{R}\}=\mathbf{R} \times \mathbf{R},
$$

the projection of $H$ on $\mathfrak{A} \xi$ is just the identity projection $I$, and $\rho((0,1), \mathfrak{A} \xi)=0$. Suppose, then, that the projection $P$ of $H$ on $\mathfrak{A} \xi$ exists. Then either $\rho((0,1), \mathfrak{A} \xi)>0$ or $\rho((0,1), \mathfrak{A} \xi)<1$. In the first case, $c=0$; in the second, $c \neq 0$. Thus if $[\mathfrak{A} x]$ exists for each $x \in H$, then we can prove that

$$
\forall x \in \mathbf{R}(x=0 \vee x \neq 0),
$$

a statement constructively equivalent to the essentially nonconstructive omniscience principle LPO:

For each binary sequence $\left(a_{n}\right)_{n \geqslant 1}$, either $a_{n}=0$ for all $n$ or else there exists $n$ such that $a_{n}=1$.

It follows from this and our Theorem 1.1 that if $\rho_{\mathfrak{A} x}\left(0,-\mathfrak{A}_{1} x\right)$ exists for each $x \in H$, then we can derive LPO.

\section{ACKNOWLEDGEMENT}

This research was partially done when the author was a visiting fellow at the Isaac Newton Institute for the Mathematical Sciences, in the programme Semantics \& Syntax: A Legacy of Alan Turing. The author thanks the referees for helpful comments that improved the presentation of the paper.

\section{REFERENCES}

[1] P. Aczel and M. Rathjen: Notes on Constructive Set Theory, Report No. 40, Institut Mittag-Leffler, Royal Swedish Academy of Sciences, 2001.

[2] E. Bishop: Foundations of Constructive Analysis, McGraw-Hill, New York, 1967.

[3] E. Bishop and D.S. Bridges: Constructive Analysis, Grundlehren der Math. Wiss. 279, Springer Verlag, Heidelberg, 1985.

[4] D.S. Bridges: 'On weak operator compactness of the unit ball of $L(H)$ ', Zeit. math. Logik Grundlagen Math. 24, 493-494, 1978.

[5] D.S. Bridges, H. Ishihara: 'A definitive constructive open mapping theorem?', Math. Logic Quarterly 44, 545-552, 1998. 
[6] D.S. Bridges and L.S. Vîţă: Techniques of Constructive Analysis, Universitext, Springer Verlag, Heidelberg, 2006.

[7] D.S. Bridges, H. Ishihara, L.S. Vîţă: 'Computing infima on convex sets, with applications in Hilbert space', Proc. Amer. Math. Soc. 132(9), 2723-2732, 2004.

[8] D.S. Bridges, H. Ishihara, L.S. Vîţă: 'A new constructive version of Baire's Theorem', Hokkaido Math. Journal 35(1), 107-118, 2006.

[9] D.S. Bridges, A. Calder, W. Julian, R. Mines, and F. Richman: 'Locating metric complements in $\mathbb{R}^{n}$ ', in Constructive Mathematics (F. Richman, ed.), Springer Lecture Notes in Math. 873, 241-249, 1981.

[10] J. Dixmier: Les algèbres d'opérateurs dans l'espace hilbertien: algèbres de von Neumann, GauthierVillars, Paris, 1981.

[11] R.V. Kadison and J.R. Ringrose: Fundamentals of the Theory of Operator Algebras, Academic Press, New York, 1983 (Vol 1) and 1988 (Vol 2).

[12] P. Martin-Löf: 'An intuitionistic theory of types', in Twenty-five Years of Constructive Type Theory (G. Sambin, J. Smith, eds), 127-172, Oxford Logic Guides 36, Clarendon Press, Oxford, 1998.

[13] S. Sakai: $C^{*}$-algebras and $W^{*}$-algebras, Springer Verlag, Heidelberg, 1971.

[14] B. Spitters: 'Constructive results on operator algebras', J. Univ. Comp. Sci. 11(12), 2096-2113, 2005.

[15] D.M. Topping: Lectures on von Neumann Algebras, van Nostrand Reinhold, London 1971. 